\title{
Syndromic Surveillance for Outbreak Detection and Investigation
}

\author{
Tom Andersson*1, 2, 4, Pär Bjelkmar ${ }^{3}$, Anette Hulth ${ }^{1}$, Johan Lindh ${ }^{1}$, Stephan Stenmark ${ }^{5}$ and \\ Mikael Widerström ${ }^{6}$
}

${ }^{1}$ Swedish Institute for Communicable Disease Control (SMI), Solna, Sweden; ${ }^{2}$ National Food Agency, Uppsala, Sweden; ${ }^{3}$ Inera AB, Stockholm, Sweden; ${ }^{4}$ Stockholm University Section for Mathematical Statistics, Stockholm, Sweden; ${ }^{5}$ Västerbotten County Medical Officer, Umeå, Sweden; ${ }^{6}$ Jämtland County Medical Officer, Östersund, Sweden

\section{Objective}

For the purpose of developing a national system of outbreak surveillance, we compared local outbreak signals in three sources of syndromic data - telephone triage of acute gastroenteritis (Swedish Health Care Direct 1177), web queries about symptoms of gastrointestinal illness (Stockholm County's website for healthcare information), and OTC pharmacy sales of anti-diarrhea medication.

\section{Introduction}

A large part of the applied research on syndromic surveillance targets seasonal epidemics, e.g. influenza, winter vomiting disease, rotavirus and RSV, in particular when dealing with preclinical indicators, e.g. web traffic (Hulth et al, 2009). The research on local outbreak surveillance is more limited. Two studies of teletriage data (NHS Direct) have shown positive and negative results respectively (Cooper et al, 2006; Smith et al, 2008). Studies of OTC pharmacy sales have reported similar equivocal performance (Edge et al, 2004; Kirian and Weintraub, 2010). As far as we know, no systematic comparison of data sources with respect to multiple point-source outbreaks has so far been published (cf. Buckeridge, 2007). In the current study, we evaluated the potential of three data sources for syndromic surveillance by analyzing the correspondence between signal properties and point-source outbreak characteristics.

\section{Methods}

The extracted data streams were compared with respect to nine waterborne and foodborne outbreaks in Sweden in 2007-2011. The analysis consisted of three parts: (1) the validation of outbreak signals by comparing signal counts during outbreak and baseline periods, (2) the estimation of detection limits by modeling signal rates (signalto-case ratios), and (3) the evaluation of early warning potential by means of signal detection analysis.

\section{Results}

The four largest outbreaks generated strong and clear outbreak signals in the 1177 triage data. The two largest outbreaks produced signals in OTC sales of anti-diarrhea. No signals could be identified in the web query data. The outbreak detection limit based on triage data was about 100-1000 cases. For two outbreaks, triage data on diarrhea provided outbreak signals early on, weeks and months respectively, potentially serving the purpose of early warning.

\section{Conclusions}

The sensitivity and specificity were highest for telephone triage data on patient symptoms. It provided the most promising source of syndromic data for surveillance of point-source outbreaks. Currently, a project has been initialized to develop and implement a national system in Sweden for daily syndromic surveillance based on 1177 Health Care Direct, supporting regional and local outbreak detection and investigation.

\section{Keywords}

syndromic surveillance; outbreak detection; point-source outbreak; outbreak investigation; data analysis

\section{Acknowledgments}

The study is part of an ongoing research and development project on syndromic surveillance (SUMO) funded by the Swedish Agency for Contingency Planning (MSB).

\section{References}

Buckeridge DL. Outbreak detection through automated surveillance: a review of the determinants of detection. J Biomed Inform. 2007 Aug;40(4):370-9.

Cooper DL, Verlander NQ, Smith GE, Charlett A, Gerard E, Willocks L, O'Brien S. Can syndromic surveillance data detect local outbreaks of communicable disease? A model using a historical cryptosporidiosis outbreak. Epidemiol Infect. 2006 Feb;134(1):13-20.

Edge VL, Pollari F, Lim G, Aramini J, Sockett P, Martin SW, Wilson J, Ellis A. Syndromic surveillance of gastrointestinal illness using pharmacy over-the-counter sales. A retrospective study of waterborne outbreaks in Saskatchewan and Ontario. Can J Public Health. 2004 Nov-Dec;95(6):446-50.

Hulth A, Rydevik G, Linde A. Web queries as a source for syndromic surveillance. PLoS One. 2009;4(2).

Kirian ML, Weintraub JM. Prediction of gastrointestinal disease with over-the-counter diarrheal remedy sales records in the San Francisco Bay Area. BMC Med Inform Decis Mak. 2010 Jul 20;10:39.

Smith S, Elliot AJ, Mallaghan C, Modha D, Hippisley-Cox J, Large S, Regan M, Smith GE. Value of syndromic surveillance in monitoring a focal waterborne outbreak due to an unusual Cryptosporidium genotype in Northamptonshire, United Kingdom, June - July 2008. Euro Surveill. 2010 Aug 19;15(33):19643.

\footnotetext{
*Tom Andersson

E-mail: tom.andersson@smi.se
} 